\title{
TÉCNICA PARA SELEÇÃo dE VARIÁVEIS APLICADA À SEPARAÇÃo DE GASES
}

\section{Célia de Figueiredo Cordeiro Neves* e Mônica Maria de Abreu Mendonça Schvartzman}

Centro de Desenvolvimento da Tecnologia Nuclear, Comissão Nacional de Energia Nuclear, CP 941, 30123-970

Belo Horizonte - MG

\section{Elizabete Jordão}

Departamento de Engenharia de Sistemas Químicos e Informática, Faculdade de Engenharia Química, Universidade Estadual de Campinas, CP 6066, 13084-970 Campinas - SP

Recebido em 9/3/01; aceito em 25/7/01

\begin{abstract}
VARIABLES SEARCH TECHNIQUE APPLIED TO GAS SEPARATION. This paper describes an experimental design technique, known as variables search, developed to expose the critical variables and screen out the irrelevant ones. It is easy to learn and use and clearly dissociates the main and interactions effects from each other. An example of air separation process by pressure swing adsorption was used to demonstrate how the variables search technique works. The phases of identification of the critical variables is shown, step by step,.
\end{abstract}

Keywords: design of experiments; variables search; gas adsorption.

\section{INTRODUÇÃo}

Todo experimento envolve uma série de variáveis (ou fatores) com maior ou menor grau de importância para o processo em estudo. Uma das principais questões que surgem em trabalhos experimentais é a determinação da influência de uma ou mais variáveis sobre outra variável de interesse. Uma prática comum em laboratório é realizar os experimentos alterando uma variável de cada vez, enquanto as demais permanecem constantes. Entretanto, a maneira mais eficiente de realizar um experimento é utilizar uma abordagem científica denominada planejamento estatístico de experimentos. Nele, todas as variáveis são modificadas ao mesmo tempo, de forma cuidadosamente planejada, através de um planejamento experimental. A razão para isso é que as variáveis podem se influenciar mutuamente e o valor ideal para uma delas pode depender do valor da outra $^{1}$. Desta forma, dados apropriados são coletados em tempo e a custos mínimos.

O planejamento experimental é uma ferramenta poderosa para estudar o efeito conjunto de vários fatores sobre uma variável resposta de interesse. Uma das técnicas mais conhecidas é o planejamento fatorial, no qual estão envolvidos $k$ fatores (ou variáveis) cada um deles presente em diferentes níveis. O caso mais simples é aquele em que cada fator $k$ está presente em apenas dois níveis. Na realização de um experimento com $k$ fatores em dois níveis, são feitas $2 \mathrm{x}$ $2 \times \ldots \times 2$ ( $k$ vezes $)=2^{k}$ observações da variável resposta e, portanto, este planejamento é denominado ${ }^{2}$ experimento fatorial $2^{k}$.

No planejamento fatorial completo, cada possível combinação dos níveis dos fatores precisa ser testada para se determinar o quanto o processo ou experimento em estudo é afetado por cada variável. O número de experimentos aumenta geometricamente com o número de variáveis, de modo que este método não é usualmente prático quando estão envolvidas acima de quatro variáveis. Para evitar a realização do grande número de experimentos necessários pelo fatorial completo, podem ser utilizados outros planejamentos experimentais como o fatorial fracionado, as redes ortogonais de Taguchi e os planejamentos de Plackett e Burman. Contudo, estas alternativas são não apenas complexas como produzem, geralmente, resultados misturados, isto é, nem sempre é possível dizer se uma determinada resposta é devida a uma interação de diversas variáveis ou ao efeito de uma única variável ${ }^{3}$.

Este trabalho descreve a aplicação de uma técnica de planejamento experimental simples e de fácil aplicação, conhecida como seleção de variáveis, a qual foi desenvolvida por Dorian Shainin, e que permite dissociar com precisão os efeitos principais das interações entre as variáveis $^{3}$. Essa técnica foi aplicada na identificação das variáveis críticas para o processo de separação do ar utilizando a tecnologia PSA ("Pressure Swing Adsorption"). Os processos PSA comerciais foram desenvolvidos a partir de 1960 e encontram aplicação industrial na remoção de contaminantes de matérias primas e efluentes gasosos e na recuperação e purificação de gases industriais ${ }^{4}$.

\section{A TÉCNICA DE SELEÇÃO DE VARIÁVEIS}

A técnica de seleção de variáveis consiste de três fases sucessivas:

\section{Fase 1}

Nesta fase são listadas todas as variáveis potencialmente relevantes para o processo e atribuídos valores "bons" (+) e "ruins" (-) a cada uma. Deve-se levar em conta que:

- A lista deve incluir até mesmo aquelas variáveis que parecem triviais, pois se uma variável crítica for omitida por ter sido considerada irrelevante, todos os experimentos desta fase terão que ser repetidos.

- Deve-se colocar em primeiro lugar na lista as variáveis consideradas mais importantes, pois elas serão examinadas primeiro na Fase 2, e pode-se poupar trabalho se for verificado que as variáveis mais importantes são as do topo da lista.

- Cada nível (+) deve ser escolhido com a certeza de que levará à resposta desejada ou "boa" e cada nível (-) deve ser selecionado por levar a uma resposta inferior ou "ruim".

\footnotetext{
*e-mail: caf@cdtn.br
} 
Em seguida são realizados seis experimentos, três com todas as variáveis na posição (+) e três na posição (-), em ordem aleatória. Todos os seis experimentos precisam ser realizados para se estabelecer com um nível de confiança de $95 \%$ que as variáveis críticas foram incluídas na lista.

Se os resultados experimentais mostrarem que as respostas "boas" e "ruins" foram produzidas e com valores bem distintos, levando-se em conta o erro experimental, pode-se afirmar que a lista contém a(s) variável(eis) crítica(s) e passa-se à Fase 2. Se esses dois critérios não forem encontrados, então ou a lista ainda não contém as variáveis críticas ou os resultados "bons" e "ruins" foram invertidos, isto é, uma posição (+) na realidade produz um resultado (-) e vice-versa. Neste caso, deve-se reavaliar a lista e/ou os valores "bons" (+) e "ruins" (-) e repetir os seis experimentos.

\section{Fase 2}

Nesta fase examina-se o significado de cada variável pela realização de experimentos aos pares, nos quais cada par enfoca uma variável particular. Inicialmente, o nível (-) da variável particular é combinado com o nível (+) de todas as outras variáveis; em seguida, os valores são trocados, isto é, o nível (+) daquela variável é combinado com o nível (-) de todas as outras variáveis. Pela troca de apenas uma variável o pesquisador pode verificar os efeitos daquela variável em particular (incluindo suas interações) pois todos os outros fatores permanecem nas condições escolhidas na Fase 1.

Os resultados desta fase podem ser $^{3}$ :

- A variável resposta não é afetada quando a variável em estudo é trocada. Nesse caso, a variável particular não influencia a resposta dentro da faixa de condições testada.

- Há uma inversão completa das respostas, isto é, as respostas "boas" tornam-se "ruins" e as "ruins" tornam-se "boas", quando a variável é trocada. Esse resultado indica que a variável em questão é a única variável crítica e nenhuma outra controlará a resposta, dentro das condições testadas.

- A troca de níveis provoca alterações em uma ou nas duas respostas mas não há uma transposição inequívoca de ambas as respostas. Isto significa que a variável testada é significativa, mas sofre interação com outras variáveis.

Os experimentos aos pares são realizados até que ocorra uma completa transposição de respostas ou pelo menos duas variáveis significativas sejam identificadas.

Na sequiência desta fase são realizados mais dois experimentos para confirmação dos resultados. Ajustam-se todas as variáveis que se supõe sejam críticas de modo que seus níveis (-) sejam combinados com os níveis (+) das demais variáveis e vice-versa. Se as respostas se inverterem, as variáveis críticas foram identificadas.

\section{Fase 3}

Nesta fase, os efeitos das variáveis críticas e suas interações são quantificados através dos métodos de análise de variância, os quais são usualmente aplicados aos dados gerados pelos planejamentos fatoriais, utilizando-se programas disponíveis comercialmente.

\section{EXEMPLO DE APLICAÇÃO DA SELEÇÃO DE VARIÁVEIS}

A tecnologia PSA é uma das mais importantes técnicas de separação que utilizam o fenômeno da adsorção. Ela compõe-se de duas etapas principais: (a) adsorção, durante a qual os componentes preferencialmente adsorvidos são removidos da alimentação e (b) dessorção, durante a qual os componentes adsorvidos são removi- dos do adsorvente, regenerando-o para que possa ser usado no ciclo seguinte. $\mathrm{O}$ aspecto essencial de um processo PSA é que a dessorção é realizada através da redução da pressão total, ao invés do aumento da temperatura ou da utilização de um gás de purga ${ }^{5}$.

Uma das principais aplicações comerciais dessa tecnologia é na separação do ar, com a produção de oxigênio e/ou nitrogênio. Utiliza-se, geralmente, um adsorvente zeolítico em um sistema automatizado contendo dois ou mais leitos.

Este trabalho apresenta um exemplo de aplicação da técnica de seleção de variáveis com o objetivo de identificar as variáveis críticas do processo PSA de enriquecimento do ar em oxigênio, utilizando uma instalação de laboratório desenvolvida para este fim ${ }^{6}$. Os resultados dos testes de separação foram obtidos do trabalho de Neves ${ }^{7}$, onde se encontra, também, uma descrição detalhada do processo.

As variáveis potencialmente relevantes para o processo em estudo foram identificadas após uma extensa revisão bibliográfica e estudo do processo. Os níveis foram ajustados para se cobrir determinada região experimental, a qual se procurou tornar a mais ampla possível, porém limitada às condições da própria instalação e da prática industrial. Além disso, a escolha dos níveis foi feita de modo que, utilizando-se o nível (+) fossem obtidos resultados considerados "bons" e, com o nível (-), os resultados fossem considerados "ruins", tendo como variável resposta a concentração de oxigênio no produto. A Tabela 1 apresenta a lista de variáveis potencialmente relevantes para o processo e seus níveis.

Tabela 1. Lista de variáveis importantes para o processo

\begin{tabular}{clcc}
\hline Número & Variável & Nível (+) & Nível (-) \\
\hline 1 & Vazão de alimentação, $\mathrm{m}^{3} / \mathrm{h}$ & 0,10 & 0,40 \\
2 & Pressão de dessorção, bar & 0,07 & 0,50 \\
3 & Tempo de adsorção, s & 5 & 30 \\
4 & Pressão de adsorção, bar & 2,00 & 1,50 \\
5 & Tempo de pressurização, s & 20 & 3 \\
6 & Número de ciclos & 20 & 5 \\
7 & Condição inicial da coluna & Pressurizada & Em vácuo \\
\hline
\end{tabular}

Foram realizados 6 ensaios, 3 com todas as variáveis no nível (+) e 3 com todas as variáveis no nível (-), sendo que a ordem de execução dos mesmos foi sorteada para garantir que variáveis não controladas não interferissem nos resultados. A Tabela 2 mostra os resultados destes ensaios. Observa-se que as respostas "boas" e "ruins" foram invariavelmente obtidas e que os dois conjuntos de resposta são distintos, sem nenhuma probabilidade de superposição de respostas, mesmo levando em conta o erro experimental. Podese, então, afirmar que a lista contém a(s) variável(eis) crítica(s), passando-se à Fase 2, na qual serão selecionadas as mais importantes.

Tabela 2. Resultados da Fase 1 da seleção de variáveis

\begin{tabular}{ccc}
\hline Ensaio & Nível das variáveis & Concentração $\left(\% \mathrm{O}_{2}\right)$ \\
\hline 01 & + & 49,5 \\
02 & + & 49,3 \\
03 & - & 20,6 \\
04 & - & 20,6 \\
05 & + & 49,3 \\
06 & - & 20,8 \\
\hline
\end{tabular}

Na Fase 2 é avaliado o significado das variáveis listadas na Fase 1 com a realização de uma série de ensaios aos pares, sendo que cada par focaliza uma variável particular. Os resultados destes ensaios 
Tabela 3. Resultados da Fase 2 da seleção de variáveis

\begin{tabular}{clccl}
\hline Ensaio & Variável em estudo & Nível das variáveis & Conc. $\left(\% \mathrm{O}_{2}\right)$ & Efeito \\
\hline 07 & Vazão de aliment., $\mathrm{m}^{3} / \mathrm{h}$ &,,,,,,-+++++ & 36,3 & Parcial, tendência aos resultados $(-)$ \\
08 & &,,,,,+-----+ & 25,2 & Parcial, tendência aos resultados (+) \\
09 & Pressão de dessorção, bar &,,,,,,+-++++ & 30,7 & Parcial, tendência aos resultados $(-)$ \\
10 & &,,,,,,-+---- & 25,5 & Parcial, tendência aos resultados (+) \\
11 & Tempo de adsorção, s &,,,,,,++-++++ & 39,6 & Parcial, tendência aos resultados $(-)$ \\
12 & &,,,,,,--+---- & 29,3 & Parcial, tendência aos resultados (+) \\
13 & Pressão de adsorção, bar &,,,,,,+++-+++ & 52,5 & Nenhum \\
14 & &,,,,,,---+-- & 23,0 & Nenhum \\
15 & Tempo de pressurização, s &,,,,,,++++-++ & 40,3 & Parcial, tendência aos resultados $(-)$ \\
16 & &,,,,,,----+-- & 22,0 & Nenhum \\
17 & Número de ciclos &,,,,,,+++++-+ & 48,1 & Nenhum \\
18 & &,,,,,,-----+- & 22,0 & Nenhum \\
19 & Cond. inicial da coluna &,,,,,,++++++- & 52,1 & Nenhum \\
20 & &,,,,,,------+ & 22,1 & Nenhum \\
\hline
\end{tabular}

podem ser vistos na Tabela 3. Três das sete variáveis, vazão de alimentação, pressão de dessorção e tempo de adsorção, tiveram um nítido efeito sobre a variável resposta. Por exemplo, no ensaio 7 a vazão de alimentação está no nível (-) e todas as demais variáveis, no nível (+). A concentração de oxigênio no produto diminuiu para $36,3 \%$, enquanto que, na Fase 1, o resultado médio do nível (+) foi de $49,4 \%$. No ensaio 8 , invertem-se os níveis, isto é, a vazão de alimentação está no nível (+) e todas as demais variáveis no nível (-). Esta alteração provocou um aumento na concentração de oxigênio no produto, passando de $20,7 \%$ (resultado médio da Fase 1) para $25,2 \%$. Uma análise semelhante pode ser feita para as variáveis pressão de dessorção e tempo de adsorção.

Nos ensaios 13 e 14 foi estudada a variável pressão de adsorção. Os resultados não mostraram nenhum efeito desta variável sobre o processo, levando-se em conta o erro experimental, quando se comparam os valores $52,5 \%$ e 23,0\% com os resultados da Fase 1: 49,4\% e $20,7 \%$, respectivamente. O mesmo tipo de análise aplica-se aos ensaios 17 a 20.

No ensaio 15 quando o tempo de pressurização estava no nível (-) e as outras variáveis no nível (+), houve uma nítida diminuição na concentração de oxigênio, enquanto que, quando as posições dos níveis foram invertidas, nenhum efeito foi observado.

Para verificar se a vazão de alimentação, a pressão de dessorção e o tempo de adsorção são realmente as variáveis críticas, foram realizados dois experimentos adicionais de verificação. Conforme mostrado na Tabela 4, houve uma completa inversão dos valores de concentração de oxigênio em relação às condições estabelecidas na Fase 1, comparando-se os ensaios 21 e 22 com as respostas $49,4 \%$ e $20,7 \%$, respectivamente, de modo que estas são, de fato, as variáveis críticas do processo.

Tabela 4. Confirmação dos resultados da seleção de variáveis

\begin{tabular}{ccc}
\hline Ensaio & Nível das variáveis & Conc. $\left(\% \mathrm{O}_{2}\right)$ \\
\hline 21 &,,,,,,---++++ & 22,9 \\
22 &,,,,,,+++---- & 41,5 \\
\hline
\end{tabular}

\section{CONCLUSÕES}

Este exemplo de aplicação ilustra a técnica de seleção de variáveis e a economia em experimentação que ela proporciona. Com apenas 22 ensaios foi possível identificar as variáveis críticas, quando teriam sido necessários 128 ensaios para a execução de um planejamento fatorial completo. A seleção de variáveis também proporciona resultados melhores do que os planejamentos fatoriais fracionários por causa do confundimento que ocorre nos mesmos, isto é, nem sempre é possível dizer se uma determinada resposta é devida a uma interação de diversas variáveis ou ao efeito de uma única variável. Além disso, como alguns dos experimentos são duplicados, o erro experimental pode ser determinado. Esta técnica representa, portanto, uma ferramenta poderosa para utilização em projetos de pesquisa e desenvolvimento.

\section{AGRADECIMENTOS}

Os autores agradecem o apoio financeiro da FAPEMIG e do programa RHAE/CNPq Meio Ambiente.

\section{REFERÊNCIAS}

1. Barros Neto, B.; Scarminio, I.S.; Bruns, R.E.; Planejamento e Otimização de Experimentos; Universidade Estadual de Campinas, Campinas, 1995, p. 14.

2. Werkema, M.C.C.; Aguiar, S.; Otimização Estatística de Processos: como Determinar a Condição de Operação de um Processo que Leva ao Alcance de uma Meta de Melhoria, Série Ferramentas da Qualidade; Fundação Christiano Ottoni: Belo Horizonte, 1996, v. 9, p. 27.

3. Sutton, N. L.; Chem. Eng. 1997, 104, 106.

4. Yang, R.T.; Gas separation by adsorption processes; Butterworths: Boston, 1987, p. 7.

5. Ruthven, D.M., Farooq, S.; Knaebel, K.S.; Pressure Swing Adsorption, VCH: New York, 1994, p. 1.

6. Figueiredo, C.A., Schvartzman, M.M.A.M., Luz, I.O.; Soares, A. E. G.; $3^{\circ}$ Encontro Nacional de Aplicações Nucleares, Águas de Lindóia, Brasil, 1995.

7. Neves, C. F. C.; Tese de Doutorado; Universidade Estadual de Campinas, Brasil, 2000. 